\title{
MORPHOLOGICAL CHANGES IN LUNGS, HEART AND LIVER CAUSED BY EXPERIMENTAL ASSOCIATED CHEST AND THIGHS TRAUMA
}

\author{
M. M. Khudobiak', M. I. Marushchak', L. M. Holovatiuk'2, T. V. Datsko'1 \\ 'I. HORBACHEVSKY TERNOPIL STATE MEDICAL UNIVERSITY, TERNOPIL, UKRAINE \\ ${ }^{2}$ TARAS SHEVCHENKO REGIONAL HUMANITARIAN PEDAGOGICAL ACADEMY OF KREMENETS, \\ KREMENETS, UKRAINE
}

Background. According to preliminary results obtained, the development of hypoxemia caused by chest trauma affected lipid peroxidation stimulation leading to disruption of cell membranes structure and consequently cell death closing the 'vicious' circle, which in our opinion and according to the literature cause multiple organ failure.

Objective. The aim of our study was to determine the features of morphological changes in cases of heart, lungs, liver injury after associated chest and both thighs trauma in rats.

Methods. For histological examination the pieces of left ventricle tissues of heart, lungs and liver were used, which were fixed in 10\% neutral formalin solution, dehydrated and embedded in paraffin blocks.

Results. It was established that associated trauma of chest and both thighs in 1 day after the injury is accompanied by disturbances of hemodynamics in parenchymal organs due to venous congestion that occurs during the development of cardiovascular disease caused by traumatic shock. In the most severe period of maximum likelihood of complications, within 3 days, respiratory hypoxia, cardiovascular disorders, metabolic acidosis, and induced programmed cell death may develop.

Conclusions. On the background of increasing pulmonary oedema (thickening of inter air sac membranes) caused by associated trauma of chest and both thighs in rats after only one day of the experiment hemodynamic changes in heart (low-blood supply of vessels in epicardium and isolated blood vessels of venous type) and liver (mainly plethora of blood vessels) were observed, which lead to morphological changes of myocardium within 3 days.

KEY WORDS: trauma; lungs; liver; heart; morphological changes.

\section{Introduction}

Trauma is the main cause of death among people of all ages, with most injuries that can be prevented by adequate quality diagnosis and correction. Over the past decade, due to increasing urbanization and mechanization of life, injuries have become an urgent social and economic problem. Multiple injuries, especially in road accidents, according to their frequency and consequences of social significance, are very important in the context of nosology forms leading to death and disability of victims and reduce labour resources of the country $[1,2]$. The level of injury in the regions of Ukraine is growing steadily, but from injuries in 200140

Corresponding author: Marya Marushchak, Department of Functional Diagnostics and Clinical Pathophysiology, I. Horbachevsky Ternopil State Medical University, 1 Maidan Voli, Ternopil, Ukraine, 46001

Phone number: +380979901202

E-mail: marushchak@tdmu.edu.ua thousand people died, and 2.3 million were injured. According to the State Statistics Committee of Ukraine, mortality, because of accidents, poisonings and injuries, takes the third place, and because of only injuries - the fourth among all causes $[3,4]$.

In recent years, a steady trend towards complications of traumatic injuries is evidenced, most of them are multiple and combined with the inherent nature of mutual burdening syndrome [5]. Combined traumatic injuries due to their complexity are especially dangerous and provide the significant threat to human life. [6] They are still unsolved problem for the world of medical science and health [7].

From the pathophysiological point of view, the risk of trauma is due to the involvement of target organs in pathological process that contributes to the development of multiple organ failure. Thus, according to Pasternak VN, it is 
established that in multiple trauma, liver becomes involved in pathological process due to reduced activity of enzyme systems and the kidneys. The author suggests that these two organs play a great part in organ failure $[8,9]$. In the literature Prydruha SM and Gasyuk NV analysed the published data regarding the mechanisms of tissue damage to various organs in different periods of traumatic disease [10]. Kozak DV and Volkov KS noted that damage to organs and tissues serves as morphological substrate of traumatic disease [11]. According to the obtained preliminary results, the development of hypoxemia, caused by chest trauma, affected lipid peroxidation stimulation that caused disruption of cell membranes structure and consequently cell death closure of 'vicious' circle, which in our and other authors opinion cause multiple organ failure with involvement of heart, lungs, liver in the pathological process [12].

The aim of our study was to determine the features of morphological changes in cases of heart, lungs, liver injury after associated chest and both thighs trauma in rats.

\section{Methods}

The study was conducted using 36 adult male white nonlinear rats of $200-220 \mathrm{~g}$ body weight. Rats were housed under standardized laboratory conditions, with $12 \mathrm{~h}$ dark/light cycle and free access to food and tab water ad libitum. All procedures were conducted according to the European Convention for the Protection of Vertebrate Animals used for Experimental and Other Scientific Purposes (Strasbourg, 1986) and General Ethical Principles of Experiments Using Animals (First National Congress of Bioethics, Kyiv, 2001). The animals were randomly divided into 3 groups: 1 control (C1) and 2 experimental (E1, E2) groups, each comprising 12 animals. The animals in the experimental group underwent thiopental sodium anaesthesia (40 mg/kg intraperitoneal) using a trocar modelled right-closed pneumothorax from fractured ribs and combined them with a broken left and right femur. Skeletal injury was simulated by applying a single shock dosed by a specially designed device on each thigh, which caused a closed fracture [13]. Impact energy was $0.375 \mathrm{~J}$, which corresponded to the injury of moderate severity. Combined injury was simulated by sequential administration of these two injuries. Mortality of animals in each group was E1(12/11), E2(12/9), E3(12/10), E4(12/10), $E 5(12 / 9)$. At the end of the experimental period, the rats were sacrificed by decapitation.
For histological examination the pieces were separated from left ventricle tissues of heart, lungs and liver and fixed in 10\% neutral formalin solution, dehydrated and embedded in paraffin blocks [14]. Then the sections, obtained by Sannomiya microtome, were stained with hematoxylin-eosin. Histological preparations were studied using light microscope and SEOSCAN and documented using the camcorder Vision CCD Camera.

\section{Results}

Histologically we have found out the beginning of morphological changes in the liver structure of experimental animals in 1 day after the experiment was established. Thus lobular structure still was intacted, sinusoid's gaps were visualized well, and some of them were a little expanded and did not contain any cellular elements. Hepatocytes were increased slightly in size, but most of them saved their organization. Their cytoplasm became enlightened, fine-grained, filled in with vacuoles of various sizes. Some nucleus grew in size, their nuclei were visualized well; the rest of the nucleus were enlightened, pycnotic corrugated (Fig. 1, 2). Area of portal tracts was increased mainly due to the plethora of vessels (Fig. 2). Perivascular oedema and lymph histiocytic infiltration were not observed.

Histological examination of the myocardium of experimental animals in 1 day after the beginning of the experiment proved low-arterial vessels blood supply in epicardium and individual vessels of venous type dilated with mild perivascular oedema. Perivascular stroma was slightly loosened because of oedema. Cardiomyocytes were visualized well; their cytoplasm

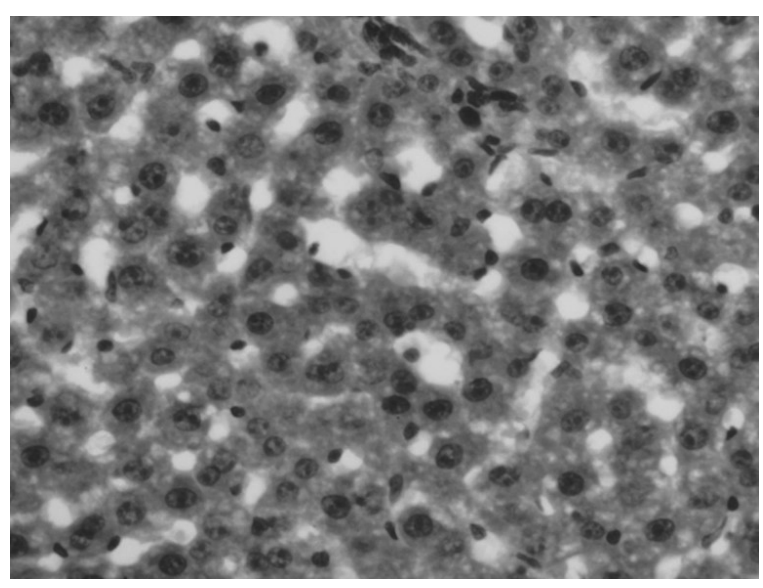

Fig. 1. Liver structure in cases of associated chest and thighs trauma on the $1^{\text {st }}$ day of the experiment. (Significant degenerative changes of hepatocytes, structural changes of nuclei). Stained with hematoxylin and eosin. $\times 200$. 
was rich, homogenous; well accumulated pigment. Nuclei were visualized in the main part of the cells. Some nuclei increased in size, karyoplasm was enlightened (Fig. 3). In pericellular spaces we detected a slight swelling, which occurred among the few red blood cells. Some of the cardiomyocytes were fragmented.

Histological examination of lungs of experimental animals revealed that in 1 day tissue blood flow was decreased. Phenomenon of dystonia with spasm of vessel walls was dominated, but land-capillary venous plethora was also observed (Fig. 4). Inter air sac membrane became thick because of a slight oedema, vascular dystonia, and focal perivascular infiltration (Fig. 5). Cellular reaction manifested by leukocytosis and macrophage infiltration. Some of alveoli were expanded; their gaps remained free of fluid. The walls of small bronchus were mainly spasmed, ciliated epithelium surface

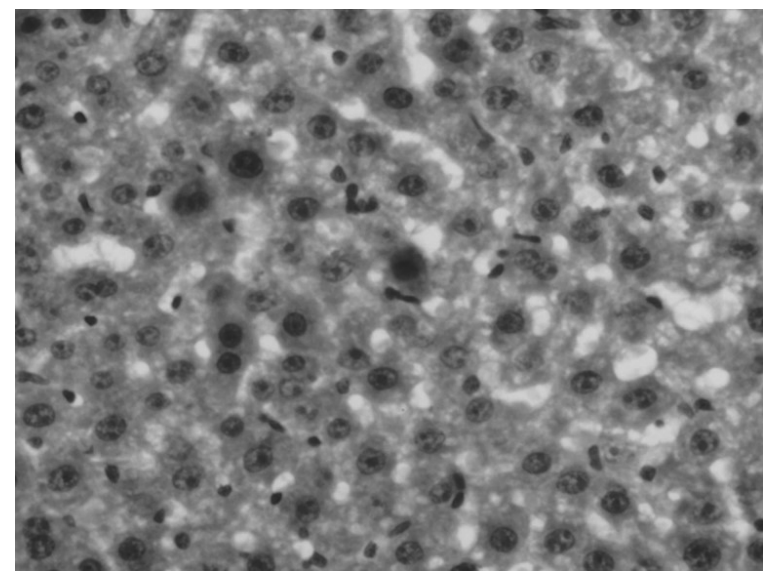

Fig. 2. Liver structure in cases of associated chest and thighs trauma in 1 day after the beginning of the experiment. (Dystrophic changes of hepatocytes, expansion sinusoid). Stained with hematoxylin and eosin. $\times 200$.

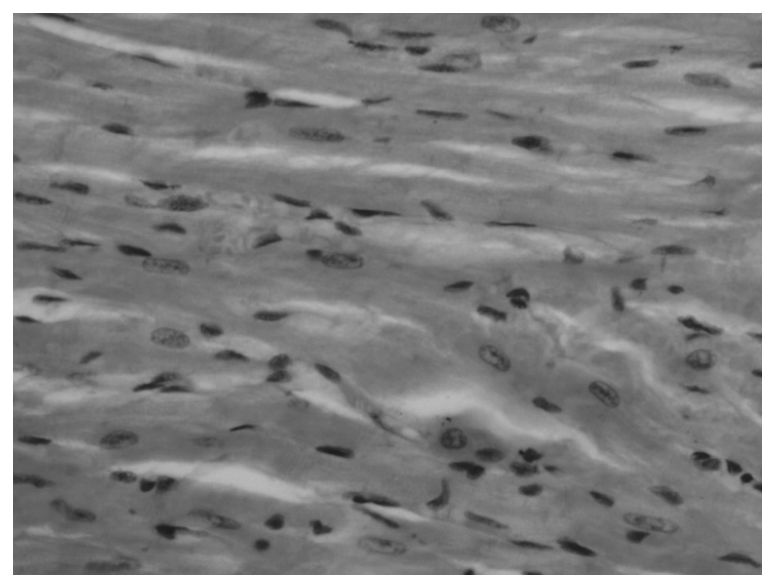

Fig. 3. Myocardium structure in cases of associated chest and thighs trauma in 1 day after the beginning of the experiment. (Slight swelling of pericellular spaces, fragmentation of some cardiomyocytes). Stained with hematoxylin and eosin. $\times 200$. became flat; in some areas pockets of inflammatory infiltration and exfoliation of the epithelium into the lumen were identified. However, the majority of gaps were free of fluid. The pulmonary pleura remained without any signs of inflammation.

Histological examination of liver of experimental animals in 3 days of the experiment proved the progression of structural changes that resulted in violation of lobular structure and disruption of cells structure. Enlightenment sinusoid was visualized poorly and practically did not contain cellular elements. Beamed organization of cells was violated; cell size was raised significantly by increasing the number of cytoplasmic inclusions. The cytoplasm was mosty enlightened, became fine-grained. The majority of nuclei were hypertrophied, nuclei were well vizualized; some of cores were enlightened, pyknotic corrugated (Fig. 5). Area

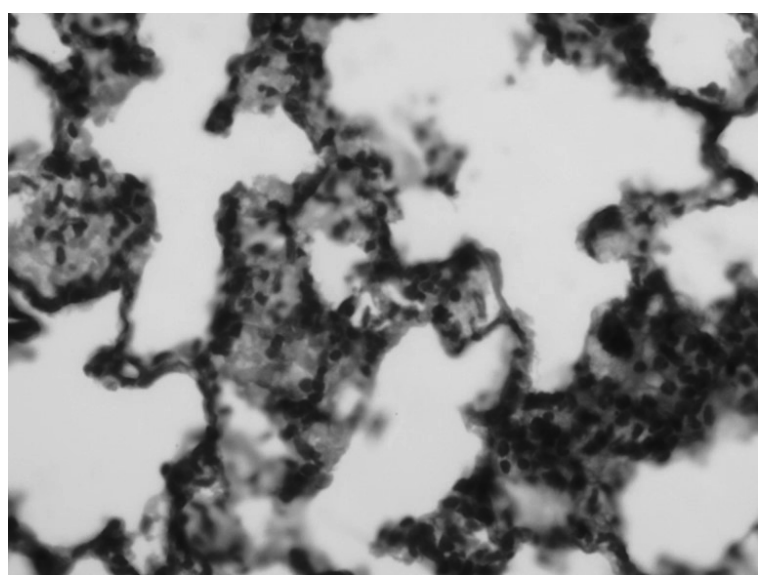

Fig. 4. Lung structure in cases of associated chest and thighs trauma in 1 day after the beginning of the experiment. (Uneven capillary venous hyperemia). Stained with hematoxylin and eosin. $\times 200$.

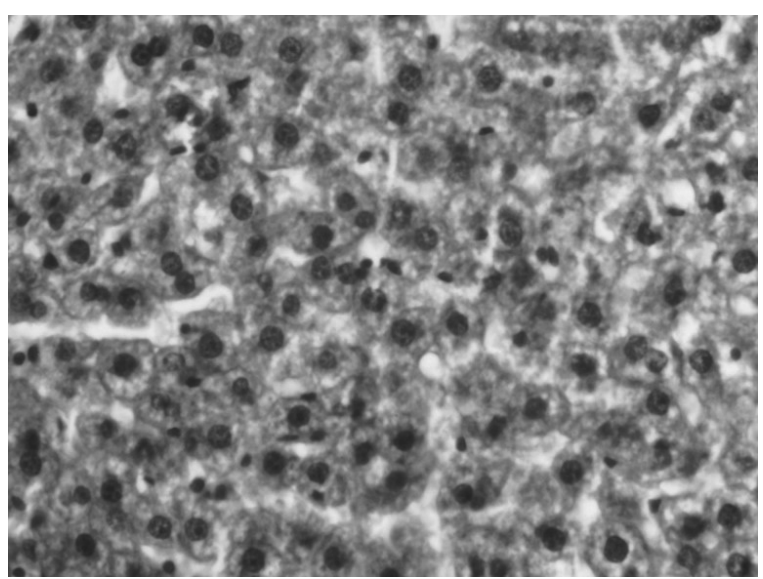

Fig. 5. Liver structure in cases of associated chest and thighs trauma in 3 days after the beginning of the experiment. (Increased dystrophic changes of hepatocytes). Stained with hematoxylin and eosin. $\times 200$. 
portal tracts were increased, mainly due to the plethora of lymph vessels and moderate histiocytic infiltration. Perivascular oedema was not observed.

Histological examination of the myocardium in 3 days of the experiment proved the increase of vessels blood supply of both arterial and venous type, their hyperemia and perivascular oedema increased. It leaded to decrease of perivasal stroma and increase of intracellular spaces (Fig. 6). The structure of the majority of cardiomyocytes changed a little. They were located evenly, their sarcoplasm remained homogeneous, cores were partly enlightened, but their contours remained clear. Only a few fibres were replated with colour that indicated of their abbreviation.

In 3 days of the experiment, the thickness of inter air sac membranes because of increasing of oedema, easing elastic and collagen fibres and focal perivascular infiltration, was observed in the lungs of rats (Fig. 7). The cell reaction was manifested by leukocytosis and moderate increase of macrophages, mast cells and lymphocytes. Enlightenment of some alveoles remained slightly enhanced, but the most of them were free of fluid. The walls of small bronchus remained spasmodic, superficial single-row ciliated epithelium became flat. In mucosa the area of focal inflammatory infiltration and exfoliation of the epithelium into the lumen increased. Pulmonary pleura inflammation was not evidenced.

\section{Discussion}

It is established that during the first 12 hours since the injury, due to local tissue damage and activation of systemic inflammatory response, acute disorders of vital functions take place $[15,16]$. The release of proteases, oxidants, leukotrienes and proinflammatory cytokines under the influence of pathogenic factors causes the damage of lung endothelium that leads to pulmonary oedema. In this study it was proved that thickening of inter air sac membranes in 3 days of the experiment and lesions of alveolar epithelium result in decrease of surfactant production and loss of normal fluid transport that impairs lung function [19, 20]. Results of the study indicate that the associated trauma of chest and both thighs in 1 day after the injury is accompanied by disturbances of hemodynamics in parenchymal organs due to venous congestion that occurs during the development of cardiovascular disease (lowblood supply in arteries of epicardium and

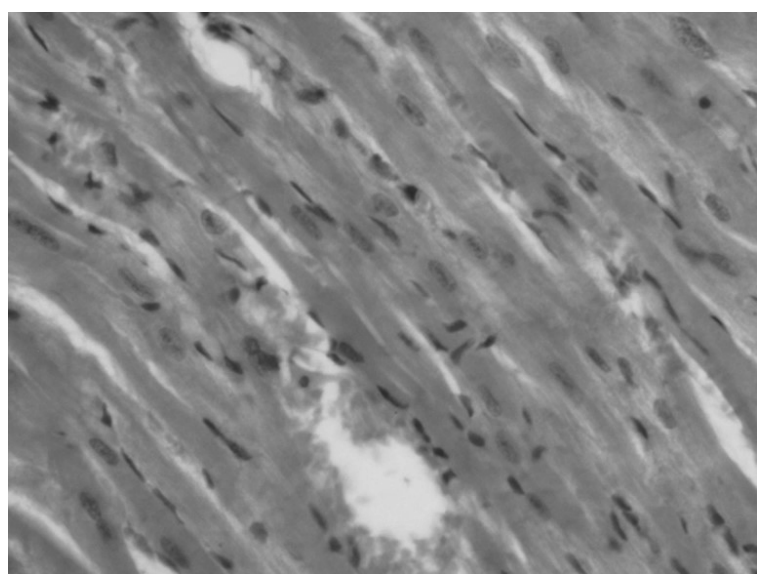

Fig. 6. Structural changes in myocardium caused by associated chest and thighs trauma in 3 days of the experiment. (Decrease of perivasal stroma and increased intracellular spaces). Stained with hematoxylin and eosin. $\times 200$.

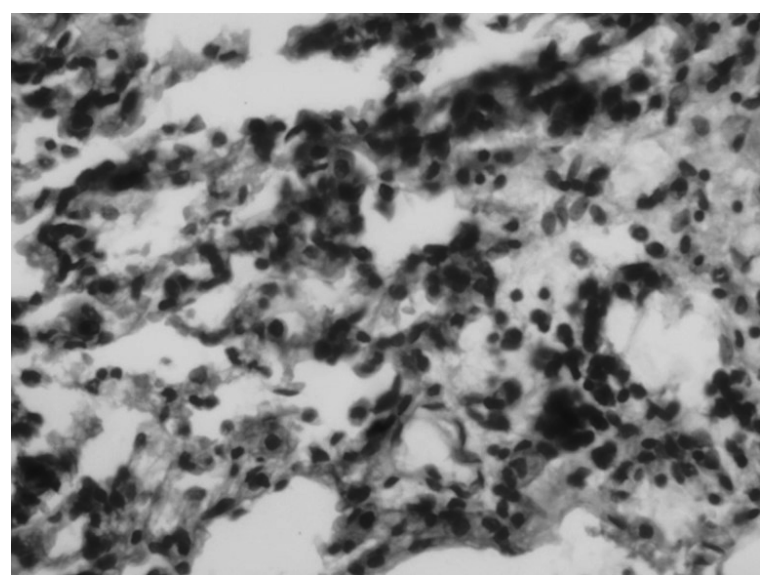

Fig. 7. Lungs structure in cases of associated chest and thighs trauma in 3 days after the beginning of the experiment. (Moderate oedema, vascular dystonia, and focal perivascular infiltration). Stained with hematoxylin and eosin. $\times 200$.

single extended venous vessels with a minor perivascular oedema) caused by traumatic shock [17]. In the lungs the system of inflammatory response becomes more active in the form of cellular reactions that are manifested by leukocytosis and macrophage infiltration.

In the most difficult period of maximum likelihood of complications, within 3 days respiratory hypoxia, cardiovascular disorders, metabolic acidosis, and induced programmed cell death may develop [18]. Previously we have found out that associated trauma of chest and both thighs in rats is accompanied by tissue hypoxia in early posttraumatic period and is characterized by the increase in the concentration of lactate and pyruvic acid from the first day of the experiment. We believe that the progressive development of hypoxia and activation of free radical oxidation and potentiate 
endogenous intoxication contract artery walls reaction and structural changes on the cellular level.

\section{Conclusions}

On the background of increasing pulmonary oedema (thickening of inter air sac membranes) provided by associated trauma of chest and both thighs in rats in only one day of the experiment, hemodynamic changes in heart (lowblood supply of vessels in epicardium and isolated blood vessels of venous type) and liver (mainly plethora of blood vessels) were observed; it leads to morphological changes of myocardium in 3 days, characterized by loosened perivasal stroma, increased intracellular spaces, and structural changes in liver: violation of lobed beam structure and hepatocytes organization, development of degenerative changes.

In the future we plan to investigate the influence of antioxidant correction on morphological changes in lungs, liver and heart after traumatic injuries of chest and hips.

\section{References}

1. Schedrenok VV, Yakovenko SE, Mohuchaya OV. Clinical and organizational aspects of combined craniocerebral injuries. St Petersburg. 2010;435.

2. Humanenko EK. Polytrauma. Actual problems and new technology in treatment. Materials of international conference: New technologies in the military and peace time surgery. 2006 April 20-22. St Petersburg. 2006;4-14.

3. Gayko GV, Kalashnikov AV, Lymar EO. State and problems of orthopedic and trauma care in Ukraine. Orthopedics, traumatology and prosthetics. 2004;2:5-9.

4. Gelfand BR. Integral systems for assessing the severity of patients with polytrauma. Bulletin of intensive care. 2004;1:58-65.

5. Berezka NI, Litovchenko VA, Garyachyy EB, et al. Optimization of surgical tactics for treating victims with polytrauma using the severity and damage severity scales. Scientific statements Bel GU. Medicine. Pharmacy. 2014;25(4):116-119.

6. Guriev CO, Tanasienko PV, Satsyk SP. Clinical and epidemiological characteristics of patients with infectious complications of trauma due to accidents. Medicine today and tomorrow. 2012;1:54.

7. Wang HE, Shapiro NI, Yealy DM. Characteristics of out-of-hospital shock care. Critical Care Medicine. 2011;39:243-245.

8. Pasternak VN. Features of biochemical homeostasis in people with severe pelvic trauma of the pelvis with an unfavorable course of traumatic illness. Archive of clinical and experimental medicine. 1997;6(30):8-15.

9. Pasternak VN, Khudobin VYu, Pasternak VV, Shpachenko NN, Chirach SH. Actual problems of the organization of assistance to victims in road traffic multiple and combined trauma. Orthopedics, traumatology and prosthetics. 2002;3:82-87.

10. Pridruga SM, Gasyuk NV. Nosotropic mechanisms of organs damage in different periods of traumatic illness. The world of medicine and biology. 2012;2:194-200.
11. Kozak DV, Volkov KS. Structural changes of some internal organs in animals with simulated polytrauma and correction with carbacetam. Scientific Bulletin of the Uzhgorod University, series Medicine. 2014;2(50):3-6.

12. Marushchak MI, Khudobiak MM, Krynytska IYa, Antonyshyn IV. Lipid peroxidation in multiple organ failure caused by associated chest and hip trauma International Journal of Medicine and Medical Research. 2016;2(2):52-55.

13. Kozak DV, inventor; Ternopil State Medical University, the patent owner. Modeling method of polytrauma. Ukraine patent number 63997. 25 Oct 2011.

14. Goralska LP, Khomich BT, Kononsky OI. Histological techniques and methods of morphological studies in normal and pathological conditions. Zhytomyr: Polissia; 2011;288.

15. Malone DL, Kuhls D, Napolitano LM, et al. Back to basics: validation of the admission systemic inflammatory response syndrome score in predicting outcome in trauma. J Trauma. 2001;51:458-463.

16. Rotstein OD. Modeling the two-hit hypothesis for evaluating strategies to prevent organ injury after shock. Resuscitation. J Trauma. 2003; 54(5):203-206.

17. Kalinkin CO, Kurapov EP, Kalinkin AO. Intensive therapy, energy and plastic provision of patients with polytrauma. The pain, anesthesia and intensive care. 2000;1:173-174.

18. Vologzhanin DA. Metabolic basis of secondary immune deficiency in traumatic disease: St Petersburg: 2005.

19. Eisner MD, Thompson BT, Schoenfeld D, et al. Acute Respiratory Distress Syndrome Network. Airway pressures and early barotraumas in patients with acute lung injury and acute respiratory distress syndrome. Am J Respir Crit Care Med. 2002;165(7):978982.

20. Marik PE. Aspiration pneumonitis and aspiration pneumonia. N Engl J Med. 2001;344(9):665-671.

\begin{tabular}{|c|c|}
\hline & \\
\hline & Received: 2017-04-06 \\
\hline ISSN 2413-6077. IJMMR 2017 Vol. 3 Issue 1 & M. M. Khudobiak et al. \\
\hline
\end{tabular}

